\title{
Editorial
}

\section{Microstructural and Microanalytical Techniques in Materials Science}

This issue of MMM is devoted to the proceedings of a 2-day Workshop which took place in Lecce (Italy) on 23-24 February 1995. It was dedicated to the memory of Professor Carmelo De Blasi, a well reknown scientist in the field of electron microscopy and a dedicated teacher, who died prematurely three years ago. To this event participated many researchers both from Italy and from abroad, who cooperated with De Blasi or had with him fruitful scientific discussions.

Twenty-eight oral contributions, including two invited lectures, were given by scientists active in the field of microstructural and microanalytical characterization of materials, by both electron and scanning probe microscopies. The first invited speaker was Professor John Steeds from HH Wills Physics Laboratory in Bristol, who presented some reflections on the work of Carmelo De Blasi and a review of recent developments in the field of convergent beam electron diffraction. The second invited speaker was Professor Ray Carpenter from Arizona State University, who reported on the investigation of the local chemistry at interfaces and boundaries in ceramics and electronic materials.

Almost all the contributions to the Lecce Workshop are reported in this volume. In overall, they give an impression of the recent, enormous developments which occurred in the field of the microstructural and microanalytical techniques, as applied to research problems in materials science.

The guest editors of this issue are particularly thankful to the members of the local organizing committee, all of whom did their thesis work under the guidance of Professor C. De Blasi (E. Carlino, M. Catalano, D. Manno and M. Re) and to the international board of referees for their rapid and efficient work. The workshop was held under the auspices of the Italian Society of Electron Microscopy (SIME).

Aldo Armigliato

CNR-Istituto LAMEL

Bologna
Lorenzo Vasanelli

Department of Materials Science

University of Lecce 